\title{
Benign Uterine Corpus PEComa
}

National Cancer Institute

\section{Source}

National Cancer Institute. Benign Uterine Corpus PEComa. NCI Thesaurus. Code C127071.

A benign neoplasm with perivascular epithelioid cell differentiation arising from the uterine corpus. It is characterized by the absence of pleomorphism and scarcity or absence of mitotic figures. It usually affects perimenopausal women. Patients present with a pelvic mass or abnormal bleeding. 